\title{
On the Kummer conjecture
}

\author{
by \\ YAming Lu and Wenpeng Zhang (Xi'an)
}

1. Introduction. Let $q$ be an odd prime, and let $h_{q}$ and $h_{q}^{+}$be the class numbers of $\mathbb{Q}\left(\zeta_{q}\right)$ and $\mathbb{Q}\left(\zeta_{q}+\zeta_{q}^{-1}\right)$ respectively $\left(\zeta_{q}=e^{2 \pi i / q}\right)$. It is well known that $h_{q}^{+} \mid h_{q}$. The famous Kummer conjecture states that

$$
h_{q}^{-}=h_{q} / h_{q}^{+} \sim 2 q\left(\frac{q}{4 \pi^{2}}\right)^{(q-1) / 4} \quad(\text { as } q \rightarrow \infty)
$$

is equivalent to

$$
\prod_{\chi(-1)=-1} L(1, \chi) \sim 1 \quad(\text { as } q \rightarrow \infty),
$$

where $\chi$ runs through Dirichlet characters modulo $q$. This conjecture has not been proved yet, but there are several works on the upper bound of $h_{q}^{-}$; for example, by using elementary methods, Feng Keqin [1] proved that

$$
h_{q}^{-}<2 q\left(\frac{q-1}{31.997158 \ldots}\right)^{(q-1) / 4},
$$

and in [2] it is mentioned that the following results have been proved:

$$
\begin{gathered}
\frac{2}{3}<\prod_{\chi(-1)=-1} L(1, \chi)<\frac{3}{2} \quad(\text { for } 5 \leq q \leq 523), \\
\frac{e^{-12.93}}{L q^{1 / 2}(\log q)^{4}}<\prod_{\chi(-1)=-1} L(1, \chi)<e^{15.49} L(\log q)^{5} \quad(\text { for any } q)
\end{gathered}
$$

with $L=e^{4.66 / \log q}$. In [4], M. Ram Murty and Yiannis N. Petridis proved the following weak Kummer conjecture: There exists a positive constant $c$

2000 Mathematics Subject Classification: Primary 11M20.

Key words and phrases: Kummer conjecture, Dirichlet $L$-function, bound estimate.

This work is supported by N.S.F. (No.10601039) of P.R. China. 
such that for almost all primes $q$,

$$
c^{-1} \leq \prod_{\chi(-1)=-1} L(1, \chi) \leq c .
$$

In this paper, we shall prove the following

TheOREM 1. For every sufficiently large prime $q$ with $q \equiv 1(\bmod 4)$,

$$
\begin{gathered}
\prod_{\chi(-1)=-1} L(1, \chi) \geq e^{-1.4}(\log q)^{-4 / 3}(\log \log q)^{-1}\left(1+O\left(\frac{1}{\log \log q}\right)\right), \\
\prod_{\chi(-1)=-1} L(1, \chi) \leq e^{0.84}(\log q)^{7 / 6}(\log \log q)\left(1+O\left(\frac{1}{\log \log q}\right)\right),
\end{gathered}
$$

where $\chi$ runs through Dirichlet characters modulo $q$.

Using the same method, we can prove the following conclusions:

THEOREM 2. Let $q$ be any sufficiently large prime. Assume that there is no exceptional zero for $L(s, \chi)$, where $\chi$ is any Dirichlet character modulo $q$. Then we have the same estimates as in Theorem 1.

THEOREM 3. Assuming the Generalized Riemann Hypothesis (GRH), for every sufficiently large prime $q$ we have

$$
\begin{gathered}
\prod_{\chi(-1)=-1} L(1, \chi) \geq e^{-2.1}(\log q)^{-4 / 3}\left(1+O\left(\frac{1}{\log q}\right)\right), \\
\prod_{\chi(-1)=-1} L(1, \chi) \leq e^{1.53}(\log q)^{7 / 6}\left(1+O\left(\frac{1}{\log q}\right)\right) .
\end{gathered}
$$

THEOREM 4. For any fixed $\varepsilon>0$, there is a positive number $Q$, which depends only on $\varepsilon$, such that if $q$ is a prime greater than $Q$, we have

$$
e^{-1.4} q^{-\varepsilon}(\log q)^{-1 / 3} \leq \prod_{\chi(-1)=-1} L(1, \chi) \leq e^{0.84} q^{\varepsilon}(\log q)^{1 / 6}
$$

The following symbols will be used in the proof of these theorems. For $q \geq 3,(l, q)=1,1 \leq l<q$, we write

$$
\begin{aligned}
\pi(x ; q, l) & =\sum_{\substack{p \leq x \\
p \equiv l(\bmod q)}} 1, \quad \theta(x ; q, l)=\sum_{\substack{p \leq x \\
p \equiv l(\bmod q)}} \log p, \\
\psi(x ; q, l) & =\sum_{\substack{n \leq x \\
n \equiv l(\bmod q)}} \Lambda(n), \quad \psi(x ; \chi)=\sum_{n \leq x} \Lambda(n) \chi(n), \\
\psi(x) & =\sum_{n \leq x} \Lambda(n), \quad x_{1}=q^{\log \log q}, \quad x_{2}=e^{q},
\end{aligned}
$$

where $\Lambda(n)$ is the von Mangoldt function. 


\section{Some lemmas}

Lemma 1. For every prime $q \geq 3$,

$$
\sum_{\chi(-1)=-1} \sum_{p>x_{2}} \frac{\chi(p)}{p} \ll q^{2} e^{-c_{1} \sqrt{q}},
$$

where $\chi$ runs through Dirichlet characters modulo $q$, and $c_{1}>0$ is a constant independent of $q$.

Proof. For an arbitrary number $y>x_{2}$, by using the identity

$$
\sum_{\chi(-1)=1} \chi(n)= \begin{cases}\frac{1}{2}(q-1) & \text { if } n \equiv \pm 1(\bmod q), \\ 0 & \text { otherwise }\end{cases}
$$

we have

$$
\begin{aligned}
\sum_{\chi(-1)=-1} \sum_{x_{2}<p \leq y} \frac{\chi(p)}{p} & =\sum_{x_{2}<p \leq y} \frac{1}{p} \sum_{\chi(-1)=-1} \chi(p) \\
& =\frac{q-1}{2}\left(\sum_{\substack{x_{2}<p \leq y \\
p \equiv 1(\bmod q)}} \frac{1}{p}-\sum_{\substack{x_{2}<p \leq y \\
p \equiv-1(\bmod q)}} \frac{1}{p}\right) \\
& =\frac{q-1}{2} \int_{x_{2}}^{y} \frac{1}{u} d\{\pi(u ; q, 1)-\pi(u ; q,-1)\} .
\end{aligned}
$$

The Siegel-Walfisz theorem yields

$$
\begin{aligned}
\sum_{\chi(-1)=-1} \sum_{x_{2}<p \leq y} \frac{\chi(p)}{p} & \ll q e^{-c_{1} \sqrt{\log x_{2}}}+q \int_{x_{2}}^{y} e^{-c_{1} \sqrt{\log u}} \frac{d u}{u} \\
& \ll q e^{-c_{1} \sqrt{\log x_{2}}}+q e^{-c_{1} \sqrt{\log x_{2}}} \log x_{2} \ll q^{2} e^{-c_{1} \sqrt{q}} .
\end{aligned}
$$

Since $y$ is arbitrary, we can easily get (6) by letting tend $y$ to infinity in the last formula.

Lemma 2 (see $[5, \S 17.1]$ ). Assume $q \geq 3$ is any integer and $s=\sigma+i t$. Then there is at most one character $\chi$ modulo $q$ such that the function $L(s, \chi)$ has a zero in the region

$$
\sigma \geq 1-\frac{c_{2}}{\log (q(|t|+2))}
$$

where $c_{2}$ is a positive constant. If such an exceptional function exists, the corresponding character $\tilde{\chi}$ must be a nonprincipal real character modulo $q$, and $L(s, \widetilde{\chi})$ has only one zero $\widetilde{\beta}$ (this zero must be a real zero) in the above region.

Lemma 3 (see [5, Theorem 33.3.1]). If the exceptional zero $\widetilde{\beta}$ in Lemma 2 exists, then there are positive constants $c_{3}, c_{4}$ such that $\widetilde{\beta}$ is the only zero 
of $\prod_{\chi \bmod q} L(s, \chi)$ in the region

$$
\left\{\begin{array}{l}
\sigma \geq 1-\frac{c_{3}}{\log (q(|t|+2))} \log \frac{c_{4} e}{\widetilde{\delta} \log (q(|t|+2))}, \\
\widetilde{\delta} \log (q(|t|+2)) \leq c_{4}
\end{array}\right.
$$

where $\widetilde{\delta}=1-\widetilde{\beta}$.

Lemma 4 (see [5, Theorem 33.2.8]). Assume $q \geq 3$ is any integer and $\chi$ is a Dirichlet character modulo $q$. Denote by $N(\alpha, T, \chi)$ the number of zeros of $L(s, \chi)$ in the region $\alpha \leq \sigma \leq 1,|t| \leq T$, and write $N(\alpha, T, q)=$ $\sum_{\chi \bmod q} N(\alpha, T, \chi)$. Then for any $T \geq 2$ and $1 / 2 \leq \alpha \leq 1$, we have

$$
N(\alpha, T, q) \ll(q T)^{3(1-\alpha)} .
$$

Lemma 5 (see [5, Theorem 18.1.5]). Assume $x \geq 2, T \geq 2, q \geq 3$. Then for every nonprincipal character $\chi$ modulo $q$,

$$
\psi(x, \chi)=-\widetilde{E} \frac{x^{\widetilde{\beta}}}{\widetilde{\beta}}-\sum_{|\gamma| \leq T}^{\prime} \frac{x^{\varrho}}{\varrho}+O\left(\frac{x \log ^{2}(x q T)}{T}+\log ^{2}(x q)+\widetilde{E} x^{1 / 4}\right),
$$

where

$$
\widetilde{E}= \begin{cases}1, & \chi=\widetilde{\chi} \\ 0, & \chi \neq \widetilde{\chi}\end{cases}
$$

$\tilde{\chi}$ is the exceptional character that possibly exists, and $\sum^{\prime}$ is the sum over all nontrivial zeros $\varrho=\beta+i \gamma$ of $L(s, \chi)$ except the exceptional zeros $\widetilde{\beta}$ and $1-\widetilde{\beta}$.

Lemma 6. Assume $x \geq 2, T \geq 2, q \geq 3$ is a prime number, and $l$ is a positive integer satisfying $1 \leq l<q$. Then

$$
\begin{aligned}
\theta(x ; q, l)= & \frac{\psi(x)}{q-1}-\frac{\widetilde{E}(q) \widetilde{\chi}(l)}{q-1} \cdot \frac{x^{\widetilde{\beta}}}{\widetilde{\beta}}-\frac{1}{q-1} \sum_{\chi \neq \chi^{0}} \bar{\chi}(l) \sum_{|\gamma| \leq T}^{\prime} \frac{x^{\varrho}}{\varrho} \\
& +O\left(\frac{x \log ^{2}(x q T)}{T}+\log ^{2}(x q)+x^{1 / 2}\right)
\end{aligned}
$$

where

$$
\widetilde{E}(q)= \begin{cases}1 & \text { if the exceptional character } \widetilde{\chi} \bmod q \text { exists } \\ 0 & \text { otherwise. }\end{cases}
$$

Proof. We have

$$
\psi(x ; q, l)=\sum_{n \leq x} \frac{\Lambda(n)}{\phi(q)} \sum_{\chi \bmod q} \bar{\chi}(l) \chi(n)=\frac{1}{q-1} \sum_{\chi \bmod q} \bar{\chi}(l) \psi(x, \chi) .
$$


Since $q$ is a prime number, we easily get

$$
\begin{aligned}
\psi\left(x, \chi^{0}\right) & =\sum_{\substack{n \leq x \\
(n, q)=1}} \Lambda(n)=\psi(x)-\sum_{n \leq x, q \mid n} \Lambda(n) \\
& =\psi(x)-\sum_{q^{m} \leq x} \log q=\psi(x)+O(\log x) .
\end{aligned}
$$

Combining this with Lemma 5, we have

$$
\begin{aligned}
\psi(x ; q, l)= & \frac{\psi(x)}{q-1}+\frac{1}{q-1} \sum_{\chi \neq \chi^{0}} \bar{\chi}(l) \psi(x, \chi)+O\left(\frac{1}{q} \log x\right) \\
= & \frac{\psi(x)}{q-1}-\frac{\widetilde{E}(q) \widetilde{\chi}(l)}{q-1} \cdot \frac{x^{\widetilde{\beta}}}{\widetilde{\beta}}-\frac{1}{q-1} \sum_{\chi \neq \chi^{0}} \bar{\chi}(l) \sum_{|\gamma| \leq T}^{\prime} \frac{x^{\varrho}}{\varrho} \\
& +O\left(\frac{x \log ^{2}(x q T)}{T}+\log ^{2}(x q)+\widetilde{E}(q) \frac{x^{1 / 4}}{q}\right)
\end{aligned}
$$

which proves the lemma by using $\theta(x ; q, l)=\psi(x ; q, l)+O\left(x^{1 / 2}\right)$.

LEMMA 7. Let $A=\min \left(c_{2}, c_{3}, c_{4}\right)$, where $c_{2}, c_{3}, c_{4}$ are defined in Lemmas 2 and 3. Then for every sufficiently large integer $q$, we have

$$
\sum_{\chi \bmod q} \sum_{|\gamma| \leq T}^{\prime} u^{\beta-1} \ll\left(\frac{u}{(q T)^{3}}\right)^{-A / \log (q T)}+u^{-1 / 2} q T \log (q T)
$$

for $u \geq x_{1}=q^{\log \log q}$ and $T=q^{4} ; \sum^{\prime}$ and $\beta$ are defined in Lemma 5 .

Proof. From Lemmas 2 and 3, we have:

(i) If the exceptional zero $\widetilde{\beta}$ exists and satisfies $\widetilde{\delta} \log (q T) \leq A$, then $\prod_{\chi \bmod q} L(s, \chi)$ does not vanish in the region

$$
\sigma \geq 1-\frac{A}{\log (q T)} \log \frac{A e}{\widetilde{\delta} \log (q T)}, \quad|t| \leq T
$$

except at $s=\widetilde{\beta}$.

(ii) If $\widetilde{\delta} \log (q T)>A$ or the exceptional zero does not exist, then $\prod_{\chi \bmod q} L(s, \chi)$ does not vanish in the region $\sigma \geq 1-A / \log (q T)$, $|t| \leq T$.

Hence if we choose

$$
\eta_{0}=A \log \frac{A e}{\delta_{0} \log (q T)}
$$

where

$$
\delta_{0}= \begin{cases}\widetilde{\delta}, & \widetilde{\delta} \log (q T) \leq A \\ A / \log (q T), & \widetilde{\delta} \log (q T)>A \text { or the exceptional zero does not exist }\end{cases}
$$


then $\eta_{0} \geq A$, and $\prod_{\chi \bmod q} L(s, \chi) \neq 0$ in the region $\sigma \geq 1-\eta_{0} / \log (q T)$, $|t| \leq T$ except at one point $s=\widetilde{\beta}$. Hence

$$
\sum_{\chi \bmod q} \sum_{|\gamma| \leq T}^{\prime} u^{\beta-1} \ll \sum_{\chi \bmod q} \sum_{\substack{|\gamma| \leq T \\ \beta \geq 1 / 2}}^{\prime} u^{\beta-1}=-\int_{1 / 2}^{1-\eta_{0} / \log (q T)} u^{\alpha-1} d_{\alpha} N(\alpha, T, q) .
$$

Making use of $N(1 / 2, T, q) \ll q T \log (q T)$ and Lemma 4, we obtain

$$
\begin{aligned}
\sum_{\chi \bmod q} \sum_{|\gamma| \leq T}^{\prime} u^{\beta-1} & \ll \int_{1 / 2}^{1-\eta_{0} / \log (q T)} N(\alpha, T, q) u^{\alpha-1} \log u d \alpha+u^{-1 / 2} q T \log (q T) \\
& \ll(\log u) \int_{1 / 2}^{1-\eta_{0} / \log (q T)}\left(\frac{u}{(q T)^{3}}\right)^{\alpha-1} d \alpha+u^{-1 / 2} q T \log (q T) \\
& \ll \frac{\log u}{\log u-3 \log (q T)}\left(\frac{u}{(q T)^{3}}\right)^{-\eta_{0} / \log (q T)}+u^{-1 / 2} q T \log (q T),
\end{aligned}
$$

and (12) follows at once from the choice of $u, T$ and $\eta_{0} \geq A$.

Lemma 8. For every sufficiently large prime $q \equiv 1(\bmod 4)$, we have

$$
\sum_{\substack{\chi \bmod q \\ \chi(-1)=-1}} \sum_{x_{1}<p \leq x_{2}} \frac{\chi(p)}{p} \ll(\log q)^{-A / 5}(\log \log q)^{-1},
$$

where $A$ is defined in Lemma 7.

Proof. Making use of (7) and Lemma 6, we have

$$
\begin{aligned}
& \sum_{\substack{\chi \bmod q \\
\chi(-1)=-1}} \sum_{x_{1}<p \leq x_{2}} \frac{\chi(p)}{p}=\sum_{x_{1}<p \leq x_{2}} \frac{1}{p} \sum_{\chi(-1)=-1} \chi(p) \\
= & \frac{q-1}{2}\left(\sum_{\substack{x_{1}<p \leq x_{2} \\
p \equiv 1(\bmod q)}} \frac{1}{p}-\sum_{\substack{x_{1}<p \leq x_{2} \\
p \equiv-1(\bmod q)}} \frac{1}{p}\right) \\
= & \frac{q-1}{2} \int_{x_{1}}^{x_{2}} \frac{1}{u \log u} d\{\theta(u ; q, 1)-\theta(u ; q,-1)\} \\
= & \int_{x_{1}}^{x_{2}} \frac{1}{u \log u} d\left\{\frac{\widetilde{E}(q)}{2}(\widetilde{\chi}(-1)-1) \frac{u^{\widetilde{\beta}}}{\widetilde{\beta}}-\frac{1}{2} \sum_{\chi \neq \chi^{0}}(1-\bar{\chi}(-1)) \sum_{|\gamma| \leq T}^{\prime} \frac{u^{\varrho}}{\varrho}\right\} \\
& +O\left(\frac{q \log ^{2}\left(x_{2} q T\right)}{T \log x_{2}}+\frac{q}{\sqrt{x_{1}} \log x_{1}}+\int_{x_{1}}^{x_{2}}\left(\frac{u q \log ^{2}(u q T)}{T}+u^{1 / 2} q\right) \frac{\log ^{2} u+1}{u^{2} \log ^{2} u} d u\right) .
\end{aligned}
$$


Notice that $q$ is a prime and $\tilde{\chi}$ is nonprincipal real character, so that

$$
\widetilde{\chi}(-1)=\left(\frac{-1}{q}\right)=(-1)^{(q-1) / 2}=1,
$$

where $\left(\frac{n}{q}\right)$ is the Legendre symbol modulo $q$. Hence

$$
\begin{aligned}
& \sum_{\substack{\chi \bmod q \\
\chi(-1)=-1}} \sum_{x_{1}<p \leq x_{2}} \frac{\chi(p)}{p}=-\int_{x_{1}}^{x_{2}}\left(\sum_{\chi(-1)=-1} \sum_{|\gamma| \leq T}^{\prime} u^{\varrho-1}\right) \frac{d u}{u \log u} \\
& \quad+O\left(\frac{q \log ^{2}\left(x_{2} q T\right)}{T \log x_{2}}+\frac{q}{\sqrt{x_{1}} \log x_{1}}+\int_{x_{1}}^{x_{2}}\left(\frac{u q \log ^{2}(u q T)}{T}+u^{1 / 2} q\right) \frac{\log u+1}{u^{2} \log ^{2} u} d u\right) \\
& =-\int_{x_{1}}^{x_{2}}\left(\sum_{\chi(-1)=-1} \sum_{|\gamma| \leq T}^{\prime} u^{\varrho-1}\right) \frac{d u}{u \log u}+O\left(\frac{q \log ^{2}(q T) \log ^{2} x_{2}}{T}+\frac{q}{\sqrt{x_{1}} \log x_{1}}\right) .
\end{aligned}
$$

If we choose $T=q^{4}$ and make use of Lemma 7 as well as the definition of $x_{1}, x_{2}$, we obtain

$$
\begin{aligned}
\sum_{\substack{\chi \bmod q \\
\chi(-1)=-1}} \sum_{x_{1}<p \leq x_{2}} \frac{\chi(p)}{p} & \ll \int_{x_{1}}^{x_{2}}\left(\sum_{\chi \bmod q} \sum_{|\gamma| \leq T}^{\prime} u^{\varrho-1}\right) \frac{d u}{u \log u}+\frac{\log ^{2} q}{q} \\
& \ll \int_{x_{1}}^{x_{2}}\left\{\left(\frac{u}{q^{15}}\right)^{-A / 5 \log q}+u^{-1 / 2} q^{5} \log q\right\} \frac{d u}{u \log u}+\frac{\log ^{2} q}{q} \\
& \ll \int_{x_{1}}^{x_{2}} u^{-1-A / 5 \log q} \frac{d u}{\log u}+\frac{\log ^{2} q}{q} \\
& \ll \frac{1}{\log x_{1}} \cdot \frac{5 \log q}{A} x_{1}^{-A / 5 \log q}+\frac{\log ^{2} q}{q} \\
& \ll(\log q)^{-A / 5}(\log \log q)^{-1} .
\end{aligned}
$$

This completes the proof of the lemma.

Lemma 9 (see [5, Theorem 28.6.1]). Assume $(q, l)=1$ and $1 \leq l<q<$ $y \leq x$. Then

$$
\pi(x ; q, l)-\pi(x-y ; q, l)<\frac{2}{\phi(q)} \cdot \frac{y}{\log (y / q)} .
$$

Lemma 10. Let $q \geq 3$ be a prime number, $1 \leq l<q, l$ is not a prime, and $x \geq q^{2}$. Then

$$
\sum_{\substack{p \leq x \\ p \equiv l(\bmod q)}} \frac{1}{p} \leq \frac{2}{q-1}\left(\log \log \frac{x}{q}+\frac{1}{2}+\frac{1}{\log q}\right) .
$$


Proof. Using Abel's identity, we obtain

$$
\sum_{\substack{2 q<p \leq x \\ p \equiv l(\bmod q)}} \frac{1}{p}=\frac{1}{x} \pi(x ; q, l)-\frac{1}{2 q} \pi(2 q ; q, l)+\int_{2 q}^{x} \frac{1}{u^{2}} \pi(u ; q, l) d u .
$$

If we choose $x=y>q$ in Lemma 9 , we have

$$
\pi(x ; q, l)<\frac{2}{\phi(q)} \cdot \frac{x}{\log (x / q)} \quad(x>q) .
$$

Combining this with (16), we find that

$$
\begin{aligned}
\sum_{\substack{2 q<p \leq x \\
p \equiv l(\bmod q)}} \frac{1}{p} \leq \frac{1}{x} \cdot \frac{2}{q-1} \cdot \frac{x}{\log (x / q)}+\int_{2 q}^{x} \frac{1}{u^{2}} \cdot \frac{2}{q-1} \cdot \frac{u}{\log (u / q)} d u \\
\leq \frac{2}{q-1} \log \log \frac{x}{q}+\frac{2}{q-1} \cdot \frac{1}{\log q}
\end{aligned}
$$

we have used $x \geq q^{2}$ in the last inequality. Since $l$ is not a prime, we have

$$
\sum_{\substack{p \leq 2 q \\ p \equiv l(\bmod q)}} \frac{1}{p} \leq \frac{1}{q}
$$

and we easily deduce (15) from the discussion above.

Lemma 11. Assume $q$ is a sufficiently large prime with $q \equiv 1(\bmod 4)$, and define

Then

$$
\sum_{1}=\sum_{\substack{\chi \bmod q \\ \chi(-1)=-1}} \sum_{p} \frac{\chi(p)}{p} .
$$

$$
\left|\sum_{1}\right| \leq \log \log q+\log \log \log q+\frac{1}{2}+O\left(\frac{1}{\log \log q}\right) .
$$

Proof. From (7) we get

$$
\begin{aligned}
\sum_{\chi(-1)=-1} \sum_{p \leq x_{1}} \frac{\chi(p)}{p} & =\sum_{p \leq x_{1}} \frac{1}{p} \sum_{\substack{\chi(-1)=-1 \\
2}(p)}\left(\sum_{\substack{p \leq x_{1} \\
p \equiv 1(\bmod q)}} \frac{1}{p}-\sum_{\substack{p \leq x_{1} \\
p \equiv-1(\bmod q)}} \frac{1}{p}\right) .
\end{aligned}
$$

Thus

$$
-\frac{q-1}{2} \sum_{\substack{p \leq x_{1} \\ p \equiv-1(\bmod q)}} \frac{1}{p} \leq \sum_{\chi(-1)=-1} \sum_{p \leq x_{1}} \frac{\chi(p)}{p} \leq \frac{q-1}{2} \sum_{\substack{p \leq x_{1} \\ p \equiv 1(\bmod q)}} \frac{1}{p}
$$

If we choose $x=x_{1}, l=1$ and $x=x_{1}, l=q-1$ respectively in Lemma 10, 
and put the results into (18), we obtain

$$
\begin{aligned}
\left|\sum_{\chi(-1)=-1} \sum_{p \leq x_{1}} \frac{\chi(p)}{p}\right| & \leq \log \log \frac{x_{1}}{q}+\frac{1}{2}+\frac{1}{\log q} \\
& \leq \log \log q+\log \log \log q+\frac{1}{2}+\frac{1}{\log q} .
\end{aligned}
$$

Applying Lemmas 1 and 8 yields (17).

LEMMA 12. Let $q$ be a sufficiently large prime, and define

$$
\sum_{2}=\sum_{\substack{\chi \bmod q \\ \chi(-1)=-1}} \sum_{p} \sum_{j=2}^{\infty} \frac{\chi\left(p^{j}\right)}{j p^{j}}
$$

Then

$$
\begin{aligned}
& \sum_{2} \geq-\frac{1}{3} \log \log q-\frac{1}{6}\left(2+\log \frac{8}{\log ^{2} 2}+\gamma\right)+O\left(\frac{1}{\log q}\right), \\
& \sum_{2} \leq \frac{1}{6} \log \log q+\frac{1}{12}\left(2+\log \frac{2}{\log ^{2} 2}+\gamma\right)+O\left(\frac{1}{\log q}\right)
\end{aligned}
$$

where $\gamma$ is the Euler constant.

Proof. We have

$$
\begin{aligned}
\sum_{2} & =\sum_{p} \sum_{j=2}^{\infty} \frac{1}{j p^{j}} \sum_{\chi(-1)=-1} \chi\left(p^{j}\right) \\
& =\frac{q-1}{2}\left\{\sum_{p} \sum_{\substack{j=2 \\
p^{j} \equiv 1(\bmod q)}}^{\infty} \frac{1}{j p^{j}}-\sum_{p} \sum_{\substack{j=2 \\
p^{j} \equiv-1(\bmod q)}}^{\infty} \frac{1}{j p^{j}}\right\} .
\end{aligned}
$$

Since

$$
\begin{aligned}
\sum_{p} \sum_{\substack{j=2 \\
p^{j} \equiv 1(\bmod q)}}^{\infty} \frac{1}{j p^{j}} & =\sum_{p<q} \sum_{\substack{j=2 \\
p^{j} \equiv 1(\bmod q)}}^{\infty} \frac{1}{j p^{j}}+O\left(\sum_{p>q} \sum_{j=2}^{\infty} \frac{1}{j p^{j}}\right) \\
& =\sum_{p<q} \sum_{\substack{j=2 \\
p^{j} \equiv 1(\bmod q)}}^{\infty} \frac{1}{j p^{j}}+O\left(\sum_{p>q} \frac{1}{p^{2}}\right) \\
& =\sum_{p<q} \sum_{\substack{j=2 \\
p^{j} \equiv 1(\bmod q)}}^{\infty} \frac{1}{j p^{j}}+O\left(\frac{1}{q \log q}\right)
\end{aligned}
$$

and in the same way

$$
\sum_{p} \sum_{\substack{j=2 \\ p^{j} \equiv-1(\bmod q)}}^{\infty} \frac{1}{j p^{j}}=\sum_{p<q} \sum_{\substack{j=2 \\ p^{j} \equiv-1(\bmod q)}}^{\infty} \frac{1}{j p^{j}}+O\left(\frac{1}{q \log q}\right),
$$


we deduce from (21) that

$$
\sum_{2}=\frac{q-1}{2}\left(\sum_{p<q} \sum_{\substack{j=2 \\ p^{j} \equiv 1(\bmod q)}}^{\infty} \frac{1}{j p^{j}}-\sum_{p<q} \sum_{\substack{j=2 \\ p^{j} \equiv-1(\bmod q)}}^{\infty} \frac{1}{j p^{j}}\right)+O\left(\frac{1}{\log q}\right) .
$$

Let $g$ be a primitive root modulo $q, \delta(p)$ be the exponent order of $p$ modulo $q$ for $p<q$ (that is to say, $\left.\delta(p)=\min \left\{d>0: p^{d} \equiv 1(\bmod q)\right\}\right)$, and let $k(p)$ $(1 \leq k(p) \leq q-1)$ denote the integer satisfying $p \equiv g^{k(p)}(\bmod q)$ for $p<q$. Then

$$
\delta(p)=\frac{q-1}{(k(p), q-1)},
$$

and thus

$$
\begin{aligned}
\sum_{2}= & \frac{q-1}{2}\left(\sum_{p<q} \sum_{\substack{j=2 \\
k(p) j \equiv 0(\bmod q-1)}}^{\infty} \frac{1}{j p^{j}}-\sum_{p<q} \sum_{\substack{j=2 \\
k(p) j \equiv(q-1) / 2(\bmod q-1)}}^{\infty} \frac{1}{j p^{j}}\right) \\
& +O\left(\frac{1}{\log q}\right) \\
= & \frac{q-1}{2}\left(\sum_{p<q} \sum_{j=0(\bmod \delta(p))}^{\infty} \frac{1}{j p^{j}}-\sum_{\substack{p<q \\
2 \mid \delta(p)}} \sum_{\substack{j=2 \\
j \equiv(p) / 2(\bmod \delta(p))}}^{\infty} \frac{1}{j p^{j}}\right)+O\left(\frac{1}{\log q}\right) .
\end{aligned}
$$

Since $\delta(p) \geq 3$ when $p<q$ because $q$ is a sufficiently large prime, we have

$$
\begin{aligned}
& \sum_{2} \\
= & \frac{q-1}{2}\left(\sum_{p<q} \sum_{j=1}^{\infty} \frac{1}{j \delta(p) p^{j \delta(p)}}-\sum_{\substack{p<q \\
2 \mid \delta(p)}}^{\infty} \sum_{j=1}^{\infty} \frac{1}{(j-1 / 2) \delta(p) p^{(j-1 / 2) \delta(p)}}\right) \\
& +O\left(\frac{1}{\log q}\right) \\
= & \frac{q-1}{2}\left(-\sum_{p<q} \frac{1}{\delta(p)} \log \left(1-\frac{1}{p^{\delta(p)}}\right)-\sum_{p<q} \frac{1}{\delta(p)} \log \frac{1+p^{-\delta(p) / 2}}{1-p^{-\delta(p) / 2}}\right) \\
& +O\left(\frac{1}{\log q}\right) \\
= & \frac{q-1}{2}\left\{\sum_{p<q} \frac{1}{\delta(p)} \log \left(1+\frac{1}{p^{\delta(p)}-1}\right)-2 \sum_{p<q} \frac{1}{\delta(p)} \log \left(1+\frac{1}{p^{\delta(p) / 2}}\right)\right\} \\
& +O\left(\frac{1}{\log q}\right)
\end{aligned}
$$


and this implies that

$$
\begin{aligned}
\sum_{2} & \geq-(q-1) \sum_{\substack{p<q \\
2 \mid \delta(p)}} \frac{1}{\delta(p)} \log \left(1+\frac{1}{p^{\delta(p) / 2}}\right)+O\left(\frac{1}{\log q}\right) \\
& \geq-(q-1) \sum_{\substack{p<q \\
2 \mid \delta(p)}} \frac{1}{\delta(p)} \cdot \frac{1}{p^{\delta(p) / 2}}+O\left(\frac{1}{\log q}\right) .
\end{aligned}
$$

Notice that $2 \mid \delta(p)$ implies $p^{\delta(p) / 2} \equiv-1(\bmod q)$, thus $p^{\delta(p) / 2} \geq q-1$ and

$$
\frac{1}{p^{\delta(p) / 2}} \leq \frac{1}{p^{\delta(p) / 2}+1} \cdot \frac{q}{q-1},
$$

so that

$$
\sum_{2} \geq-q \sum_{\substack{p<q \\ 2 \mid \delta(p)}} \frac{1}{\delta(p)} \cdot \frac{1}{p^{\delta(p) / 2}+1}+O\left(\frac{1}{\log q}\right)
$$

On the other hand, we can also deduce from (22) that

$$
\sum_{2} \leq \frac{q-1}{2} \sum_{\substack{p<q \\ 2 \nmid \delta(p)}} \frac{1}{\delta(p)} \cdot \frac{1}{p^{\delta(p)}-1}+O\left(\frac{1}{\log q}\right) .
$$

Since $\delta(p) \geq 3$ for $p<q$, we have

$$
\begin{aligned}
& \sum_{2} \geq-\frac{q}{3} \sum_{\substack{p<q \\
2 \mid \delta(p)}} \frac{1}{p^{\delta(p) / 2}+1}+O\left(\frac{1}{\log q}\right) \\
& \sum_{2} \leq \frac{q}{6} \sum_{\substack{p<q \\
2 \nmid \delta(p)}} \frac{1}{p^{\delta(p)}-1}+O\left(\frac{1}{\log q}\right) .
\end{aligned}
$$

We write $p^{\delta(p)}=l(p) q+1$. Because $2 \mid \delta(p)$ implies $p^{\delta(p) / 2} \equiv-1(\bmod q)$, we can write $p^{\delta(p) / 2}=h(p) q-1$ if $2 \mid \delta(p)$. Then

$$
\begin{aligned}
\sum_{\substack{p<q \\
2 \nmid \delta(p)}} \frac{1}{p^{\delta(p)}-1} & =\sum_{\substack{p<q \\
2 \nmid \delta(p)}} \frac{1}{l(p) q} \\
& =\frac{1}{q}\left(\sum_{\substack{p<q \\
2 \nmid \delta(p), l(p)<q}} \frac{1}{l(p)}+\sum_{\substack{p<q \\
2 \nmid \delta(p), l(p) \geq q}} \frac{1}{l(p)}\right) \\
& =\frac{1}{q} \sum_{\substack{p<q \\
2 \nmid \delta(p), l(p)<q}} \frac{1}{l(p)}+O\left(\frac{1}{q \log q}\right) .
\end{aligned}
$$


Using the same method, we have

$$
\sum_{\substack{p<q \\ 2 \mid \delta(p)}} \frac{1}{p^{\delta(p) / 2}+1}=\frac{1}{q} \sum_{\substack{p<q \\ 2 \mid \delta(p), h(p)<q}} \frac{1}{h(p)}+O\left(\frac{1}{q \log q}\right) .
$$

Since $l(p)<q \Rightarrow \delta(p) \leq n_{1}=[(2 \log q) / \log 2]$ and $h(p)<q \Rightarrow \delta(p) \leq n_{2}=$ $[(4 \log q) / \log 2]$, we deduce from $(25),(26)$ that

$$
\begin{gathered}
\sum_{\substack{p<q \\
2 \nmid \delta(p)}} \frac{1}{p^{\delta(p)}-1} \leq \frac{1}{q} \sum_{\substack{p<q \\
2 \nmid \delta(p), \delta(p) \leq n_{1}}} \frac{1}{l(p)}+O\left(\frac{1}{q \log q}\right), \\
\sum_{\substack{p<q \\
2 \mid \delta(p)}} \frac{1}{p^{\delta(p) / 2}+1}=\frac{1}{q} \sum_{\substack{p<q \\
2 \mid \delta(p), \delta(p) \leq n_{2}}} \frac{1}{h(p)}+O\left(\frac{1}{q \log q}\right) .
\end{gathered}
$$

It is well known that for every $d \mid q-1$, there are exactly $\phi(d)$ integers in the reduced residue class modulo $q$ with $d$ as their exponent order modulo $q$, so the number of terms of the sum on the right hand side of (27) is less than

$$
\sum_{\substack{n \leq n_{1} \\ n \mid q-1,2 \nmid n}} \phi(n) \leq n_{1} \sum_{\substack{n \leq n_{1} \\ n \mid q-1,2 \nmid n}} 1 \leq \frac{1}{2} n_{1}^{2} \leq \frac{2}{\log ^{2} 2} \log ^{2} q .
$$

It is obvious that $l(p)$ is different for each $p$, and

$$
l(p) \begin{cases}\text { is even } & \text { if } p>2, \\ \geq 1 & \text { if } p=2,\end{cases}
$$

so we infer from (27) that

$$
\begin{aligned}
\sum_{\substack{p<q \\
2 \nmid \delta(p)}} \frac{1}{p^{\delta(p)}-1} \leq & \frac{1}{q}\left(1+\sum_{n \leq \frac{2}{\log ^{2} 2} \log ^{2} q} \frac{1}{2 n}\right)+O\left(\frac{1}{q \log q}\right) \\
= & \frac{1}{q}\left\{1+\frac{1}{2}\left(\log \left(\frac{2}{\log ^{2} 2} \log ^{2} q\right)+\gamma+O\left(\frac{1}{\log ^{2} q}\right)\right)\right\} \\
& +O\left(\frac{1}{q \log q}\right) \\
= & \frac{1}{q}\left(\log \log q+1+\frac{1}{2} \log \frac{2}{\log ^{2} 2}+\frac{\gamma}{2}\right)+O\left(\frac{1}{q \log q}\right) .
\end{aligned}
$$

Also, the number of terms of the sum on the right hand side of (28) is less than

$$
\sum_{\substack{n \leq n_{2} \\ n|q-1,2| n}} \phi(n) \leq \frac{1}{2} n_{2}^{2} \leq \frac{8}{\log ^{2} 2} \log ^{2} q .
$$


Because $h(p)$ is different for each $p$, and

$$
h(p) \begin{cases}\text { is even } & \text { if } p>2 \\ \geq 1 & \text { if } p=2\end{cases}
$$

we deduce from (28) that

$$
\begin{aligned}
\sum_{\substack{p<q \\
2 \mid \delta(p)}} \frac{1}{p^{\delta(p) / 2}+1} & \leq \frac{1}{q}\left(1+\sum_{n \leq \frac{8}{\log ^{2} 2} \log ^{2} q} \frac{1}{2 n}\right)+O\left(\frac{1}{q \log q}\right) \\
& =\frac{1}{q}\left(\log \log q+1+\frac{1}{2} \log \frac{8}{\log ^{2} 2}+\frac{\gamma}{2}\right)+O\left(\frac{1}{q \log q}\right) .
\end{aligned}
$$

The lemma now follows at once from (23), (24), (29), (30).

\section{Proof of the theorems}

Proof of Theorem 1. It is easy to verify that $\prod_{\chi(-1)=-1} L(1, \chi)$ is a positive real number. Making use of the identity

$$
L(1, \chi)=\prod_{p}\left(1-\frac{\chi(p)}{p}\right)^{-1} \quad\left(\chi \neq \chi^{0}\right)
$$

we have

$$
\begin{aligned}
\log \left(\prod_{\chi(-1)=-1} L(1, \chi)\right) & =\log \left(\prod_{\chi(-1)=-1} \prod_{p}\left(1-\frac{\chi(p)}{p}\right)^{-1}\right) \\
& =-\sum_{\chi(-1)=-1} \sum_{p} \log \left(1-\frac{\chi(p)}{p}\right) \\
& =\sum_{\chi(-1)=-1} \sum_{p} \sum_{j=1}^{\infty} \frac{\chi\left(p^{j}\right)}{j p^{j}}=\sum_{1}+\sum_{2},
\end{aligned}
$$

so that Lemmas 11 and 12 imply

$$
\begin{aligned}
& \log \left(\prod_{\chi(-1)=-1} L(1, \chi)\right) \geq-\frac{4}{3} \log \log q-\log \log \log q+\log A_{1}+O\left(\frac{1}{\log \log q}\right), \\
& \log \left(\prod_{\chi(-1)=-1} L(1, \chi)\right) \leq \frac{7}{6} \log \log q+\log \log \log q+\log A_{2}+O\left(\frac{1}{\log \log q}\right),
\end{aligned}
$$

with

$$
\begin{aligned}
& A_{1}=\exp \left(-\frac{1}{6}\left(5+\log \frac{8}{\log ^{2} 2}+\gamma\right)\right) \\
& A_{2}=\exp \left(\frac{1}{12}\left(8+\log \frac{2}{\log ^{2} 2}+\gamma\right)\right) .
\end{aligned}
$$


A simple calculation shows that

$$
A_{1}>e^{-1.4}, \quad A_{2}<e^{0.84},
$$

and Theorem 1 follows at once.

Proof of Theorem 2. Inspecting the proof of Lemma 8 shows that if the exceptional zero does not exist, then the assertion of Lemma 8 holds for every sufficiently large prime $q$, and therefore so does the assertion of Lemma 11. If we now invoke Lemma 12, the result follows at once.

Proof of Theorem 3. We separate $\log \left(\prod_{\chi(-1)=-1} L(1, \chi)\right)$ into two parts as in (31). Because we are assuming GRH, we have

$$
\theta(x ; q, l)=\frac{\psi(x)}{q-1}+O\left(x^{1 / 2} \log ^{2} x\right)
$$

for $x \geq 2, q \geq 3,(q, l)=1$. This implies

$$
\sum_{\chi(-1)=-1} \sum_{p>q^{3}} \frac{\chi(p)}{p} \ll \frac{\log q}{\sqrt{q}} .
$$

Choosing $x=q^{3}$ in Lemma 10 yields

$$
\sum_{\substack{p \leq q^{3} \\ p \equiv l(\bmod q)}} \frac{1}{p} \leq \frac{2}{q-1}\left(\log \log q+\frac{1}{2}+\log 2+\frac{1}{\log q}\right)
$$

for $l= \pm 1$. Similar to Lemma 11, from (34), (35) we deduce that assuming GRH, for every sufficiently large prime $q$,

$$
\left|\sum_{1}\right| \leq \log \log q+\frac{1}{2}+\log 2+O\left(\frac{1}{\log q}\right) .
$$

By using (36) and Lemma 12, we have

$$
\begin{aligned}
& \log \left(\prod_{\chi(-1)=-1} L(1, \chi)\right) \geq-\frac{4}{3} \log \log q+\log A_{3}+O\left(\frac{1}{\log q}\right), \\
& \log \left(\prod_{\chi(-1)=-1} L(1, \chi)\right) \leq \frac{7}{6} \log \log q+\log A_{4}+O\left(\frac{1}{\log q}\right),
\end{aligned}
$$

with

$$
\begin{aligned}
& A_{3}=\exp \left(-\frac{1}{6}(5+9 \log 2-2 \log \log 2+\gamma)\right) \\
& A_{4}=\exp \left(\frac{1}{12}(8+13 \log 2-2 \log \log 2+\gamma)\right)
\end{aligned}
$$


A simple calculation shows that

$$
A_{3}>e^{-2.1}, \quad A_{4}<e^{1.53},
$$

which completes the proof of Theorem 3 .

Proof of Theorem 4. The procedure is similar to the proof of Theorem 3, but now we have to use the Siegel-Walfisz theorem instead of (33). Hence, for every $\varepsilon>0$, write $x_{3}=\exp \left(q^{\varepsilon}\right)$. We have the following formula corresponding to (34):

$$
\sum_{\chi(-1)=-1} \sum_{p>x_{3}} \frac{\chi(p)}{p} \ll q^{1+\varepsilon} \exp \left(-c_{5} q^{\varepsilon / 2}\right),
$$

where $c_{5}$ is a constant dependent on $\varepsilon$. Choosing $x=x_{3}$ in Lemma 10 yields

$$
\sum_{\substack{p \leq x_{3} \\ p \equiv l(\bmod q)}} \frac{1}{p} \leq \frac{2}{q-1}\left(\varepsilon \log q+\frac{1}{2}+\frac{1}{q}\right)
$$

for $l= \pm 1$. Similar to Lemma 11, from (37), (38) we infer that for every sufficiently large prime $q$ and all $\varepsilon>0$,

$$
\left|\sum_{1}\right| \leq \varepsilon \log q+\frac{1}{2}+O\left(q^{1+\varepsilon} \exp \left(-c_{5} q^{\varepsilon / 2}\right)+\frac{1}{\log q}\right) .
$$

Combining this with Lemma 12 and (31), we conclude that for all $\varepsilon>0$ and every sufficiently large prime $q$,

$$
\begin{aligned}
\log \left(\prod_{\chi(-1)=-1} L(1, \chi)\right) \geq & -\varepsilon \log q-\frac{1}{3} \log \log q+\log A_{1} \\
& +O\left(q^{1+\varepsilon} \exp \left(-c_{5} q^{\varepsilon / 2}\right)+\frac{1}{\log q}\right), \\
\log \left(\prod_{\chi(-1)=-1} L(1, \chi)\right) \leq & \varepsilon \log q+\frac{1}{6} \log \log q+\log A_{2} \\
& +O\left(q^{1+\varepsilon} \exp \left(-c_{5} q^{\varepsilon / 2}\right)+\frac{1}{\log q}\right) .
\end{aligned}
$$

Together with (32) this completes the proof of Theorem 4.

\section{References}

[1] K. Q. Feng, On the first factor of the class number of a cyclotomic field, Proc. Math. Amer. Soc. 84 (1982), 479-482.

[2] - Introduction to Algebraic Number Theory, Shanghai Science and Technology Press, Shanghai, 1988.

[3] H. L. Montgomery and R. C. Vaughan, The large sieve, Mathematika 20 (1973), 119-134.

[4] M. R. Murty and Y. N. Petridis, On Kummer's conjecture, J. Number Theory 90 (2001), 294-303. 
[5] Chengdong Pan and Chengbiao Pan, Elements of the Analytic Number Theory, Science Press, Beijing, 1991.

[6] - - - The Elementary Number Theory, Peking Univ. Press, Beijing, 2003.

Yaming Lu

Research Center for Basic Science

Xi'an Jiaotong University

Xi'an, Shaanxi, 710049, P.R. China

E-mail: lymxjtu@163.com
Wenpeng Zhang Department of Mathematics Northwest University Xi'an, Shaanxi, 710069, P.R. China E-mail: wpzhang@nwu.edu.cn 